\title{
Fourier transform infrared (FTIR) spectroscopy for analysis of extra virgin olive oil adulterated with palm oil.
}

\begin{abstract}
Fourier transform infrared (FTIR) spectroscopy has been developed for analysis of extra virgin olive oil (EVOO) adulterated with palm oil (PO). Measurements were made on pure EVOO and that adulterated with varying concentrations of PO (1.0-50.0\% wt./wt. in EVOO). Two multivariate calibrations, namely partial least square (PLS) and principle component regression (PCR) were optimized for constructing the calibration models, either for normal spectra or its first and second derivatives. The discriminant analysis (DA) was used for classification analysis between EVOO and that adulterated with PO and the other vegetable oils (palm oil, corn oil, canola oil, and sunflower oil). Frequencies at fingerprint region, especially at 1500-1000 $\mathrm{cm}-1$, were exploited for both quantification and classification. Either PLS or PCR at first derivative spectra revealed the best calibration models for predicting the concentration of adulterated EVOO samples, with coefficient of determination (R2) of 0.999 and root mean standard error of cross validation (RMSECV) of 0.285 and 0.373 , respectively. DA was able to classify pure and adulterated samples on the basis of their FTIR spectra with no misclassified group obtained. In addition, DA was also effective enough to classify EVOO samples as the distinct group from the evaluated other vegetable oils.
\end{abstract}

Keyword: FTIR; Extra virgin oil; Palm Oil; Multivariate calibration; Discriminant analysis. 